\begin{tabular}{ccc}
\hline & & 0 \\
& International Journal of Case Reports \\
& (ISSN:2572-8776)
\end{tabular}

\title{
Invasive Haemophilus influenzae disease in Northwestern Ontario First Nations communities: Case Series
}

Chelsea J. Kubinec MSci ${ }^{1}$, Len Kelly MD MClinSci ${ }^{2}$, Sarah Byce MD, MSci ${ }^{1}$, Raymond S.W. Tsang MMedSc PhD ${ }^{3}$, Marina Ulanova MD PhD ${ }^{4}$

${ }^{1}$ Medical Student, Northern Ontario School of Medicine, Thunder Bay, Ontario

${ }^{2}$ Sioux Lookout Meno Ya Win Health Centre, Sioux Lookout, Ontario

${ }^{3}$ Vaccine Preventable Bacterial Diseases, National Microbiology Laboratory, Public Health Agency of Canada, Winnipeg, Manitoba

${ }^{4}$ Division of Medical Sciences, Northern Ontario School of Medicine, Thunder Bay, Ontario

\section{ABSTRACT}

We present clinical and microbiological data of 5 pediatric cases of invasive Haemophilus influenzae disease, which occurred over

*Correspondence to Author:

a period of 10 months in the service area of a regional hospital of Marina Ulanova

Northwestern Ontario. Four cases of invasive $H$. influenzae type Division of Medical Sciences, a (Hia) disease presented either as meningitis, non-complicated Northern Ontario School of Medand complicated pneumonia, or soft tissue infection in children between 7 months and 6 years of age. Although the cases were icine, Lakehead University, MS3006, 955 Oliver Road, Thunder from different communities with no known common exposure, the Hia isolates demonstrated similar phenotypic and genotypic characteristics. One case of invasive disease due to nontypeable

How to cite this article:

$H$. influenzae (NTHi) presented as chorioamnionitis in an adolesChelsea J. Kubinec, Len Kelly, Sarcent. The data emphasize the significance of Hia and NTHi as a cause of serious disease in Indigenous communities.

Bay, ON P7B 5E1

ah Byce, Raymond S.W. Tsang, Marina Ulanova. Invasive Haemophilus influenzae disease in Northwestern Ontario First Nations communities:

Keywords: Haemophilus influenzae, invasive disease, First NaCase Series . International Journal tions communities

of Case Reports, 2020 4:140






\section{Introduction}

In the post-Haemophilus influenzae type b (Hib) vaccine era, the prevalence of non-Hib bacteria in the etiology of invasive $\mathrm{H}$. influenzae disease has increased ${ }^{1}$. Since 2002, invasive $H$. influenzae type a (Hia) disease is consistently present in First Nations communities of Northwestern Ontario ${ }^{2,3}$. As part of our continuing surveillance of invasive $H$. influenzae disease, during the period of 10 months, from May 2018 to March 2019, we identified 5 new pediatric cases.

\section{Study setting}

The Sioux Lookout Meno Ya Win Health Centre (SLMHC) serves a population of 31,700 in Northwestern Ontario including 26 remote fly-in communities; $82 \%$ of the population is First Nations 4. Primary care and public health services are provided by in-community nurses supported by a physician who visits one week per month and provides daily telephone contact when not in community. Emergency physician telephone support is provided $24 / 7$ by the physician practice based in Sioux Lookout. Patients are triaged for treatment in community or flown to a general hospital or a tertiary care centre, depending on their severity. Serology and microbiology specimens collected in the nursing station are transported the SLMHC laboratory for processing.

\section{Methodology}

The cases of invasive $H$. influenzae disease were identified based on $\mathrm{H}$. influenzae isolation from normally sterile body sites (blood, cerebrospinal fluid). Demographic and clinical data were retrospectively collected from the SLMHC hospital charts and their primary care medical records. Identification of $H$. influenzae was done using standard methods and confirmed by $16 \mathrm{~S}$ ribosomal RNA sequencing; serotyping was performed by both bacterial agglutination test and PCR to detect serotype-specific genes; clonal analysis of $H$. influenzae isolates was done by multilocus sequencing typing (MLST) in the National Microbiology Laboratory (NML) as previously described ${ }^{3}$. Detection of $\beta$-lactamase and antibiotic susceptibility testing were performed in the
NML using DrySlide nitrocefin reagent (BBL, Becton Dickinson, Oakville, Ontario, Canada) and the disk diffusion method following the Clinical and Laboratory Standards Institute guidelines ${ }^{5}$, respectively.

The age-specific population information (as of July 31,2017$)$ for the catchment population of SLMHC was derived from provincial health card number registry data as represented in the electronic medical records.

The SLMHC Research Review and Ethics Committee and Lakehead University Research Ethics Board approved this study.

\section{Description of Cases (Table)}

Case \#1 was a 7-month-old girl who was born small for gestational age at 36 weeks, presented to a community health centre with lethargy, fever, irritability and vomiting. The patient was transferred to a tertiary care regional hospital for a full septic workup, including a lumbar puncture, which demonstrated cloudy cerebrospinal fluid, which was culture positive for Hia. Intravenous ceftriaxone and vancomycin were administered, and the infant recovered well with some mild sensorineural hearing loss, which normalized at 6-month follow up.

Case \#2 was a 17-month-old boy who presented to community health centre with fever and a cough. The child had a chest x-ray, which showed a bilateral pneumonia. Blood cultures grew Hia. Treatment with intravenous ceftriaxone took place as an outpatient in his community. Radiographic and clinical follow up data were not available.

Case \#3 was an adolescent pregnant female who presented at near-term gestation with evidence of fetal distress by electronic fetal monitoring and delivered a healthy female infant with normal APGAR scores. The pregnancy had been complicated by gestational diabetes, anemia and opioid use disorder. Maternal blood cultures were positive for nontypeable $H$. influenzae (NTHi). The infant did well post partum.

Case \#4 was a previously healthy 2-year-old girl who presented with a purulent soft tissue 
infection of the left ankle, following a 4-day history of increasing foot pain and non-ambulation. She was transferred to SLMHC for a surgical evacuation of the abscess and initiation of ceftriaxone and vancomycin. Hia was isolated from abscess and blood. She was subsequently transferred to a regional pediatrics service for assessment of her delayed postoperative ambulation. The MRI found no evidence of bone or joint involvement and she made a complete recovery.
Case \#5 was a previously healthy 6-year-old boy who presented to a community health centre with abdominal pain, fever and vomiting following a 10-day history of cough, rhinorrhea and feeling unwell. The child was transferred to a regional pediatrics service for the diagnosis and treatment of a right lower lobe pneumonia complicated by an empyema. Treatment included intravenous ceftriaxone and salbutamol. Follow up at 7 months was complete.

Table: Cases of invasive Haemophilus influenzae disease in the Sioux Lookout Meno Ya Win Health Centre catchment area from May 2018 to March 2019

\begin{tabular}{|c|c|c|c|c|c|c|c|c|c|c|c|}
\hline $\begin{array}{l}\text { Case } \\
\#\end{array}$ & Age & Sex & Ethnicity & $\begin{array}{l}\text { H. influenzae } \\
\text { isolation site }\end{array}$ & Serotype & Biotype & $\begin{array}{l}\text { Sequence } \\
\text { type }\end{array}$ & $\begin{array}{l}\text { Antibiotic sensi- } \\
\text { tivity }^{1}\end{array}$ & $\begin{array}{l}\text { Clinical presen- } \\
\text { tation/ Diagnosis }\end{array}$ & $\begin{array}{l}\text { Length of } \\
\text { hospital } \\
\text { stay (days) }\end{array}$ & $\begin{array}{l}\text { Disease out- } \\
\text { come }\end{array}$ \\
\hline 1 & $\begin{array}{l}7 \\
\text { months }\end{array}$ & $\mathrm{F}$ & First Nations & $\mathrm{CSF}^{2}$ & A & II & 929 & $\begin{array}{l}\text { Sensitive to all, } \\
\beta \text {-lactamase } \\
\text { negative }\end{array}$ & Meningitis & 16 & $\begin{array}{l}\text { Transferred } \\
\text { to tertiary } \\
\text { care centre; } \\
\text { recovered }\end{array}$ \\
\hline 2 & $\begin{array}{l}17 \\
\text { months }\end{array}$ & $M$ & First Nations & Blood & A & II & 23 & $\begin{array}{l}\text { Sensitive to all, } \\
\beta \text {-lactamase } \\
\text { negative }\end{array}$ & Pneumonia & 0 & $\begin{array}{l}\text { Outpatient } \\
\text { treatment; } \\
\text { recovered }\end{array}$ \\
\hline 3 & $\begin{array}{l}15 \\
\text { years }\end{array}$ & $\mathrm{F}$ & First Nations & Blood & $\begin{array}{l}\text { Non- } \\
\text { typeable }\end{array}$ & III & 556 & $\begin{array}{l}\text { Sensitive to all, } \\
\beta \text {-lactamase } \\
\text { negative }\end{array}$ & Chorioamnionitis & 5 & $\begin{array}{l}\text { General hos- } \\
\text { pital; recov- } \\
\text { ered }\end{array}$ \\
\hline 4 & $\begin{array}{l}2 \\
\text { years }\end{array}$ & $\mathrm{F}$ & First Nations & Blood & A & II & 23 & $\begin{array}{l}\text { Sensitive to all, } \\
\beta \text {-lactamase } \\
\text { negative }\end{array}$ & $\begin{array}{l}\text { Soft tissue infec- } \\
\text { tion (abscess), } \\
\text { bacteremia }\end{array}$ & 10 & $\begin{array}{l}\text { Transferred } \\
\text { to tertiary } \\
\text { care centre; } \\
\text { recovered }\end{array}$ \\
\hline 5 & $\begin{array}{l}6 \\
\text { years }\end{array}$ & $M$ & First Nations & Blood & $\mathrm{A}$ & II & 23 & $\begin{array}{l}\text { Sensitive to all, } \\
\beta \text {-lactamase } \\
\text { negative }\end{array}$ & $\begin{array}{l}\text { Pneumonia, em- } \\
\text { pyema }\end{array}$ & 13 & $\begin{array}{l}\text { Transferred } \\
\text { to tertiary } \\
\text { care centre; } \\
\text { recovered }\end{array}$ \\
\hline
\end{tabular}

${ }^{1}$ Antibiotic sensitivity testing was performed for ampicillin, penicillin, amoxicillin/clavulanic acid, ampicillin/sulbactam, cefaclor, cefuroxime, cefixime, ceftriaxone, cefepime, meropenem, imipenem, chloramphenicol, clarithromycin, erythromycin, trimethoprim/sulfamethoxazole, levofloxacin, sparfloxacin, tetracycline. ${ }^{2} \mathrm{CSF}$, Cerebrospinal fluid

\section{Discussion}

$H$. influenzae is a human-restricted Gram-negative bacterial pathogen, which commonly colonizes the nasopharynx and occasionally the genitourinary tract; the organism can cause local or invasive disease including otitis media, sinusitis, pneumonia, meningitis, and sepsis. Encapsulated forms of $H$. influenzae are classified into 6 serotypes (a-f) based on antigenic characteristics of their polysaccharide capsule; non-encapsulated forms are referred to as non-typeable (NTHi). H. influenzae type b (Hib) was the major cause of pediatric meningitis before the introduction of Hib conjugate vaccine ${ }^{6}$. Since routine pediatric immunization against Hib started in
Canada in the early 1990s, epidemiology of invasive $H$. influenzae disease has significantly changed, with a 95\% decline in incidence rates of Hib disease but an increase in prevalence of disease caused by non-Hib, especially NTHi strains ${ }^{1}$. During the last 2 decades, $H$. influenzae type a (Hia) has emerged as an important cause of invasive disease in some areas of North America, with highest numbers of cases found in Indigenous peoples, especially in the Arctic ${ }^{7-9}$. Since 2002, we have observed consistent presence of invasive Hia disease in First Nations communities of Northwestern Ontario. Annual average incidence rates of invasive Hia disease hospitalized to SLMHC, which serves 
these communities, were $7 / 100,000$ in 20042008 and $3.1 / 100,000$ population in $2010-2015$ 2,3 .

In this study, during a period of 10 months, between May 2018 and March 2019, we observed 5 pediatric cases of invasive $H$. influenzae disease in the SLMHC service area, including four $\mathrm{Hia}$ and one NTHi. Considering that the regional population of those $\leq 16$ years is 9,918 , the annual age adjusted incidence rates reach $60.5 / 100,000$ for all invasive $H$. influenzae disease and 48.4/100,000 for Hia. For the total population $(31,698)$, the annual incidence rates would be 18.9/100,000 and 15.1/100,000, correspondingly. These numbers are lower than the incidence rates in the Arctic, but unprecedented in the rest of Canada. In Nunavut during 20002012, incidence rates of invasive Hia disease were $274.8 / 100,000$ for children $<1$ year of age and $61.2 / 100,000$ for $1-4$ years of age ${ }^{9}$. In the southwestern region of Alaska, annual average incidence rates of invasive Hia disease in 20022011 were 72.3/100,000 in those $<5$ years of age ${ }^{7}$. Although our data represent a short observation period, and the rates of invasive disease may greatly fluctuate from year to year, the findings suggest a potential increase in the incidence of invasive Hia disease in the region. However, the available data do not support an outbreak, as all the cases occurred in different communities with no epidemiological evidence of a common source of infection. Nevertheless, all four Hia isolates are closely related phenotypically and genetically; they belong to biotype II and the same clonal complex, represented by the sequence types (ST), ST-23 and ST-929, i.e. the most common clone circulating in North America ${ }^{10}$. Our ongoing studies of nasopharyngeal carriage in the SLMHC service area found that $8.5 \%$ of healthy $3-5$-year-old First Nations children carry Hia ${ }^{11}$ implying that continuing circulation of Hia in communities is an underlying reason for consistent presence of invasive $\mathrm{Hia}$ disease.

The demographics of the cases of invasive Hia disease in the SLMHC service area is remarkably different from cases hospitalized in 2011-2018 to TBRHSC, which serves the population of Northwestern Ontario at large. Whereas the majority of TBRHSC cases ( 7 out of 9 ) were seniors and adults with significant co-morbidities, including immunocompromising conditions, two pediatric cases had risk factors for invasive bacterial disease ${ }^{12}$. In contrast to the SLMHC service area with a consistent predominance of Hia over $\mathrm{NTHi}^{2,3}, 50 \%$ of invasive $\mathrm{H}$. influenzae cases in TBRHSC were caused by NTHi ${ }^{12}$.

While in the past we observed both adult and pediatric cases of invasive Hia disease in SLMHC, in the present study, all cases were pediatric ${ }^{2,3}$. Because of the small number of cases, it is uncertain whether this trend may represent changes in the pathogen virulence. The role of risk factors in this population is also unclear. In our previous study, 5 out of 7 patients with invasive Hia disease had significant underlying medical conditions that potentially decreased immunity ${ }^{2}$. In the present study, none of the cases of invasive Hia disease had any conditions known to be associated with compromised immunity. However, two cases were observed in children below the age of 2 years, which is an essential risk factor for invasive Hia disease ${ }^{13}$. Children $\leq 2$ years do not have protective anticapsular antibodies, and the highest incidence rates of invasive Hia disease have been found in this age group ${ }^{7-9}$.

Clinical presentations of invasive Hia disease in our case series were similar to ones described in literature and included severe forms such as meningitis, bilateral pneumonia and pneumonia with empyema as well as soft tissue infection with bacteremia ${ }^{13}$. All cases required antibiotic therapy for 10 to 16 days and three required transfer to tertiary care facilities, but all recovered. All isolates were sensitive to a wide range of antibiotics and none expressed $\beta$-lactamase; this agrees with reports on rare occurrence of resistant forms among Hia circulating in Canada ${ }^{9}$. Our finding of a case of chorioamnionitis in an adolescent emphasizes the importance of NTHi in etiology of perinatal infections and the role of 
risk factors such as gestational diabetes, anemia and opioid use disorder. Although there is a high risk of unfavorable outcomes of NTHi invasive disease during pregnancy in terms of pregnancy loss, fatality, and long-term consequences ${ }^{14}$, in our case, both the baby and mother recovered without sequelae.

The First Nations communities in Northwestern Ontario are known to experience unfavorable social determinants of health, which facilitate the spread of infectious disease, i.e. overcrowded housing and lack of access to clean water ${ }^{15,16}$. Many of these regional communities are under boil water advisories ${ }^{17}$. Invasive bacterial infections are more prevalent in this population than in the rest of the country; the incidence rates of invasive group A Streptococcus and methicillinresistant Staphylococcus aureus infections are multiples of the national rates ${ }^{18-20}$. Regional chiefs documented the high rate of preventable illness with a declaration of a public health state of emergency in $2016^{21}$. These conditions likely contribute to the high incidence of Hia infections in the region.

\section{Conclusion}

These observations emphasize the significance of Hia and NTHi as a cause of serious disease in First Nations communities of Northwestern Ontario and the need for developing preventive measures such as immunization of vulnerable population groups with a new Hia conjugate vaccine under development. In more general terms, both continued surveillance of $H$. influenzae in the post-Hib vaccine era and study of risk factors of invasive disease are essential for understanding reasons for increased susceptibility to serious infections among Canadian Indigenous populations.

Acknowledgements: This study was supported by the Canadian Immunization Research Network (CIRN). We thank Michelle Shuel, Nick Nordal-Budinsky, and William Hoang for their skillful technical support.

\section{References}

1. Ulanova M, \& Tsang RSW. Invasive Haemophilus influenzae disease: changing epidemiology and host-parasite interactions in the 21st century.Infect Genet Evol 2009;9:594-605.

2. Kelly L, Tsang RSW, Morgan A, Jamieson FB, Ulanova M. Invasive disease caused by $\mathrm{Hae}$ mophilus influenzae type a in Northern Ontario First Nations communities. J Med Microbiol 2011;60:384-390.

3. Eton V, Schroeter A, Kelly L, Kirlew M, Tsang RSW, Ulanova M. Epidemiology of invasive pneumococcal and Haemophilus influenzae diseases in Northwestern Ontario, Canada, 2010-2015. IJID 2017;65:27-33.

4. Walker R, Cromarty H, Kelly L, St Pierre-Hansen N. Achieving Cultural Safety in Aboriginal Health Services: Implementation of a cross-cultural safety model in a Hospital Setting. Diversity in Health and Care 2009:6(1):11-22.

5. Clinical and Laboratory Standards Institute. Performance Standards for Antimicrobial Susceptibility Testing: Twenty-eighth Informational Supplement M100-S28. CLSI, Wayne, PA: Clinical and Laboratory Standards Institute, 2018.

6. Wenger JD. Epidemiology of Haemophilus influenzae type b disease and impact of Haemophilus influenzae type b conjugate vaccines in the United States and Canada. Pediatr Infect Dis J 1998;17(9 Suppl):S132-136.

7. Bruce MG, Zulz T, DeByle C, Singleton R, et al. Haemophilus influenzae serotype a invasive disease, Alaska, USA, 1983-2011. Emerg Infect Dis 2013;19:932-937.

8. Boisvert AA, Moore D. Invasive disease due to Haemophilus influenzae type a in children in Canada's north: a priority for prevention. Can J Infect Dis Med Microbiol 2015;26:291-292.

9. Tsang RSW, Li YA, Mullen A, et al. Laboratory characterization of invasive Haemophilus influenzae isolates from Nunavut, Canada, 2000-2012. Int J Circumpolar Health 2016;75:29798.

10. Tsang RSW, Ulanova M. The changing epidemiology of invasive Haemophilus influenzae disease: Emergence and global presence of serotype a strains that may require a new vaccine for control. Vaccine 2017;35(33):4270-5.

11. Ulanova M, Nix EB, Tsang RSW, Tan B, Le Saux N. Study of carriage of Haemophilus influenzae type $\mathrm{A}$ among children $<5$ years old: a Canadian Immunization Research Network (CIRN) Study. JAMMI 2019; 4 (s1): AMMI Canada - CAVMID Annual Conference Abstracts 2019, K05, p. 33.

12. Cerqueira A, Byce $S$, Tsang RSW, Jamieson FB, Kus JV, Ulanova M. Continuing surveillance of invasive Haemophilus influenzae disease in northwestern Ontario emphasizes the importance of serotype a and non-typeable strains as causes of serious disease: a Canadian Immunization 
Research Network (CIRN) Study. Can J Microbiol 2019;65(11):805-813.

13. Ulanova M, and Tsang RSW. Haemophilus influenzae serotype a as a cause of serious invasive infections. Lancet Infect Dis 2014;14:70-82.

14. Collins S, Ramsay M, Slack MP. Risk of invasive Haemophilus influenzae infection during pregnancy and association with adverse fetal outcomes. JAMA 2014;311(11):1125-32.

15. Kovesi T. Respiratory disease in Canadian First Nations and Inuit children. Paediatr Child Health 2012;17:376-380.

16. Hennessy TW, Ritter T, Holman R, et al. The relationship between in-home water service and the risk of respiratory tract, skin, and gastrointestinal tract infections among rural Alaska Natives. Am J Public Health 2008;98:2072-8.

17. http://www.nan.on.ca/article/bill-s11-the-safedrinking-water-for-first-nations-act-457.asp Nishinaabe Aski Nation. Bill S-11: The Safe Drinking Water for First Nations Act (Accessed March 23, 2020).

18. Bocking N, Matsumoto $C$, Loewen $K$, et al. High incidence of Invasive Group A Streptococcus disease in NW Ontario First Nations communities. Open Forum Infect Dis 2017;4(1): doi.org/10.1093/ofid/ofw243.

19. Kirlew M, Rea S, Muileboom J, et al. An Invasive community-associated methicillin resistant Staphyloccus aureus: a two-year prospective study. CJRM 2014;19(3):99-102.

20. Muileboom J, Hamilton M, Parent $\mathrm{K}$, et al. Community-associated methicillin-resistant Staphylococcus aureus in northwest Ontario: a five-year report of incidence and antibiotic resistance. Can $\mathrm{J}$ Infect Dis Med Microbiol 2013;24:e42-4.

21. http://www.nan.on.ca/upload/documents/comms2016-02-24declaration-health-emerg.pdf Chief's Committee on Health, Nishnawbe Aki Nation. Declaration of a health and public health emergency in Nishnawbe Aski Nation (NAN) territory and the Sioux Lookout region: code blue order. (accessed 2016 July 25). 